\title{
Fase da vida marcada pela idade avançada: a atuação do enfermeiro na visita domiciliar
}

\author{
Life stage marked by Advanced Age: The role of nurses in home visits
}

Fase de la vida marcada por la edad avanzada: el papel de las enfermeras en las visitas domiciliarias

\section{Iasmim Moreira Sacchi de Mello ${ }^{1 *}$, Sebastião Jorge da Cunha Gonçalves ${ }^{2}$, Eliara Adelino da Silva ${ }^{3}$, Manoela Alves ${ }^{4}$ Margarida Maria Donato Dos Santos ${ }^{5}$, Mônica de Almeida Carreiro ${ }^{6}$ \\ Como citar esse artigo. de Mello, IMS; Gonçalves, SJC; da Silva, EA; Alves, M;

\author{
Resumo
} dos Santos, MMD; Carreiro, MA. Fase da vida marcada pela idade avançada: a atuação do enfermeiro na visita domiciliar. Revista Pró-UniverSUS. 2021 Jul./Dez.; 12 (2): 62 - 66.

A visita domiciliar é uma forma de atendimento à saúde focada no paciente e na família, identificando os problemas apresentados por ambos, e ao ser realizada, deve-se ter uma percepção diferenciada sobre os processos de envelhecimento e adoecimento, e planejar estratégias e cuidados que melhor se adequem às necessidades de cada indivíduo. Posto isto, este estudo possui como objetivo identificar se o enfermeiro presta uma assistência eficaz nas visitas domiciliares e os benefícios que traz para a vida do paciente. Trata-se de uma Reflexão Teórica com abordagem qualitativa baseada na revisão literária de artigos científicos através da busca pela base de dados Scientific Eletonic Library Online (Scielo), e interpretação de documentos e protocolos. $\mathrm{O}$ envelhecimento, antes considerado um fenômeno, hoje, faz parte da realidade da maioria das sociedades, e espera-se que em 2050 existam cerca de dois bilhões de pessoas com sessenta anos e mais no mundo. Como consequência do processo de envelhecimento, surgem nessa parcela da população problemas de saúde inerentes à vulnerabilidade da pessoa idosa. Sendo assim, conclui-se que a presença do enfermeiro é fundamental, ao prestar uma assistência mais eficiente durante a visita domiciliar, de modo a promover a saúde e aumentar a qualidade de vida da população idosa.

Palavras-chave: Idoso; Enfermagem; Visita domiciliar.

\begin{abstract}
The home visit is a form of health care focused on the patient and the family, identifying the problems presented by both, and when carrying out it, one must have a different view on the aging and illness processes, and carry out health planning. strategies and care that best suit each one's needs. That said, this study aims to identify whether the nurse provides effective assistance in home visits and the benefits it brings to the patient's life. It is a Theoretical Reflection with a qualitative approach based on the literary review of scientific articles from the search for the Scientific Eletonic Library Online database (Scielo), and interpretation of documents and protocols. Aging, previously considered a phenomenon, is now part of the reality of most societies, and it is estimated that in 2050 there are about two billion people aged sixty and over in the world. As a consequence of the aging process, health problems inherent to the elderly person's vulnerability arise in this portion of the population. Thus, it is concluded that the presence of nurses is essential, when providing more efficient assistance during home visits, in order to promote health and increase the quality of life of the elderly population.
\end{abstract}

Keywords: Treatment Adherence, Nurse; Home visit.

${ }^{1 *}$ Acadêmica do Curso de Enfermagem, Universidade de Vassouras. RJ, Brasil. E-mail: iasmimsacchi@gmail.com ORCID: https://orcid.org/0000-0002-6088-3146

${ }^{2}$ Mestre. Docente do Curso de Enfermagem, Universidade de Vassouras. RJ, Brasil. E-mail: sjcunha@uol.com.br ORCID: https://orcid.org/0000-0002-4228-4641

${ }^{3}$ Mestre. Docente do Curso de Enfermagem, Universidade de Vassouras. RJ, Brasil. eliaraadelinos@uol.com.br ORCID: https://orcid.org/0000-0003-4387-6662

${ }^{4}$ Mestre. Docente do curso de Enfermagem, Universidade de Vassouras. RJ, Brasil. Email: alves.manoela@gmail.com ORCID: https://orcid.org/0000-0003-4239-9577

${ }_{5}^{5}$ Doutora em Ciência da Saúde. Docente do Curso de Enfermagem, Universidade de Vassouras. RJ, Brasil. Email: familiadonato@terra.com.br ORCID: https://orcid.org/0000-00018681-5582

${ }^{6}$ Doutora em Enfermagem. Docente do Curso de Enfermagem, Univerdade de Vassouras. RJ, Brasil. Email: monica.carreiro@hotmail.com ORCID: https://orcid.org/0000000315946491 . 


\section{Resumen}

La visita domiciliaria es una forma de atención de la salud centrada en el paciente y la familia, identificando los problemas que presentan ambos, y al realizarla se debe tener una visión diferente de los procesos de envejecimiento y enfermedad, y realizar una planificación sanitaria. estrategias y cuidados que mejor se adapten a las necesidades de cada uno. Dicho esto, este estudio tiene como objetivo identificar si la enfermera brinda una asistencia eficaz en las visitas domiciliarias y los beneficios que aporta a la vida del paciente. Se trata de una Reflexión Teórica con enfoque cualitativo basado en la revisión literaria de artículos científicos a partir de la búsqueda de la base de datos Scientific Eletonic Library Online (Scielo), e interpretación de documentos y protocolos. El envejecimiento, antes considerado un fenómeno, es ahora parte de la realidad de la mayoría de las sociedades, y se estima que en 2050 hay alrededor de dos mil millones de personas de sesenta y más años en el mundo. Como consecuencia del proceso de envejecimiento, en este segmento de la población surgen problemas de salud inherentes a la vulnerabilidad del anciano. Así, se concluye que la presencia de enfermeras es fundamental, a la hora de brindar una asistencia más eficiente durante las visitas domiciliarias, a fin de promover la salud y mejorar la calidad de vida de la población anciana.

Palabras clave: Anciano; Enfermería; Visita domiciliaria.

\section{Introdução}

O envelhecimento, antes considerado um fenômeno, hoje, pertence à realidade da maioria das sociedades, e espera-se que em 2050 existam cerca de dois bilhões de pessoas com sessenta anos e mais no mundo, sendo a maioria moradora de países que se encontram em desenvolvimento ${ }^{1}$, calculando-se que existam, atualmente, cerca de 17,6 milhões de pessoas idosas ${ }^{1}$. Como consequência desse processo de envelhecimento e da vulnerabilidade do idoso, os problemas relacionados à saúde aumentam. Portanto, é importante que no serviço de Atenção Básica (AB) avaliação global do idoso seja feita, a realçar a funcionalidade.

Neste cenário, é fundamental ter a presença do/a enfermeiro/a, pois caracteriza-se como uma atenção diferenciada na arte de cuidar. $\mathrm{O}$ enfermeiro da Estratégia de Saúde da Família poderá prestar uma assistência mais eficiente ao fazer a visita domiciliar (forma de atenção domiciliar voltada ao atendimento do indivíduo, da família ou coletividade, prestada nos domicílios ou junto aos diversos recursos sociais locais, visando maior equidade de assistência em saúde), que ao ser realizada, tem como intuito: constatar, orientar, educar, fornecer, e acompanhar os casos clínicos.

Conforme o Sistema Único de Saúde (SUS), a finalidade primordial da política nacional de saúde da pessoa idosa é recuperar, manter e promover a autonomia e a independência dos idosos, obtendo medidas sendo em grupo ou individual de saúde, é considerado idoso e alvo dessas políticas todo homem ou mulher que possui 60 anos ou mais.

Assim, o enfermeiro, ao realizar o atendimento domiciliar, pela sua aproximação dos familiares e cuidados, deve-se ter uma percepção diferenciada sobre os processos de envelhecimento e adoecimento, possibilitando assim, um maior entendimento sobre o contexto domiciliar de cada indivíduo e o planejamento de estratégias e cuidados que melhor se adequem às necessidades de cada indivíduo.

Objetiva-se identificar se o enfermeiro presta uma assistência eficaz nas visitas domiciliares e os benefícios que traz para a vida do paciente.

\section{Materiais e Métodos}

Trata-se de uma Reflexão Teórica Reflexiva, com abordagem qualitativa baseada na revisão literária de artigos científicos através de busca pelas bases de dados Scientific Eletonic Library Online (Scielo), LILACS e BIREME. Além disso, foi utilizada também a interpretação de guia e protocolos nacionais relacionados ao assunto.

A busca de artigos na literatura foi realizada com os descritores: Fase da Vida; Idoso; Enfermeiro; Atenção Primária; Enfermagem, sendo delimitado o recorte temporal para os artigos do ano de 2014 até o ano de 2019. Após o levantamento dos artigos e busca exploratória, foram selecionados 6 artigos e 1 tese para composição do estudo, sendo todos de publicação nacional e analisados com o intuito de coletar informações acerca da visita domiciliar e principalmente a atuação do enfermeiro.

\section{Resultados e Discussão}

Após a seleção dos artigos e materiais como portarias e cadernos do Ministério da Saúde, foi realizada uma leitura reflexiva dos artigos, e foi permitido identificar as seguintes temáticas, a serem discutidas: processo envelhecimento, visita domiciliar, atuação do enfermeiro na visita domiciliar, vantagens da visitar domiciliar e capacitação do enfermeiro para realização da visita.

\section{Processo de Envelhecimento}


A Organização Pan-Americana de Saúde (OPAS) define envelhecimento como:

[...] um processo sequencial, individual, acumulativo, irreversível, universal, não patológico, de deterioração de um organismo maduro, próprio a todos os membros de uma espécie, de maneira que o tempo o torne menos capaz de fazer frente ao estresse do meio-ambiente e, portanto, aumente sua possibilidade de morte. ${ }^{1}$

O envelhecimento populacional é uma resposta à mudança de alguns indicadores de saúde, especialmente a queda da fecundidade e da mortalidade e o aumento da esperança de vida. Não é homogêneo para todos os seres humanos, sofrendo influência dos processos de discriminação e exclusão associados ao gênero, à etnia, ao racismo, às condições sociais e econômicas, à região geográfica de origem e à localização de moradia. ${ }^{1}$

Este pode se apresentar de duas formas, sendo uma a senescência, que consiste na diminuição progressiva da reserva funcional dos indivíduos e na senilidade, causado por determinada condição patológica que acometeu o indivíduo, fazendo-o necessitar de assistência.

Segundo a Política Nacional da Pessoa Idosa, idoso é todo indivíduo com 60 anos ou mais e conforme a estimativa realizada pelo Instituto Brasileiro de Geografia e Estatística em 2018, até o ano de 2047, a população do país deverá crescer, chegando a 233,2 milhões de pessoas. Como consequência desse processo de envelhecimento e da vulnerabilidade do idoso, os problemas relacionados à saúde aumentam. Portanto, é importante que nos serviços de atenção primária seja feita uma avaliação ampliada, enfatizando a funcionalidade. ${ }^{2}$

Além disso, acrescenta-se, ao considerar a atenção primária como porta de entrada ao sistema de saúde e como responsável pela resolução de grande parte dos problemas, por meio das tecnologias de menor densidade tecnológica, considerando o vínculo, a responsabilização e a longitudinalidade do cuidado, que se espera que a saúde do idoso seja acompanhada e considerada nas suas múltiplas dimensões. ${ }^{3}$

\section{Visita Domiciliar}

\section{A Estratégia de Saúde da Família:}

visa à reorganização da atenção básica no País, de acordo com os preceitos doSistema Único de Saúde, eétidapelo Ministério da Saúde e gestores estaduais e municipais, representados respectivamente pelo Conass e Conasems, como estratégia de expansão, qualificação e consolidação da atenção básica por favorecer uma reorientação do processo de trabalho com maior potencial de aprofundar os princípios, diretrizes e fundamentos da atenção básica, de ampliar a resolutividade e impacto na situação de saúde das pessoas e coletividades, além de propiciar uma importante relação custo-efetividade. ${ }^{4}$ vida da população e intervir nos fatores que colocam a saúde em risco, como má alimentação, falta de atividade física e hábitos de vida não saudáveis. A ESF fortalece como uma porta de entrada do Sistema único de Saúde e permite que a equipe de Saúde conheça a família, gerando uma maior adesão ao tratamento oferecido pela unidade de saúde, fazendo com que a qualidade de vida seja maior.

Dentre os instrumentos de assistência da ESF, encontra-se a visita domiciliar (VD), a qual é realizada pelos profissionais que estão inseridos na Atenção Básica, em Estratégias de Saúde da Família (ESF). É uma forma de atendimento à saúde, voltada para o foco em domicílio, evidenciando a família e a comunidade como um todo, e é considerada como:

[...] a atividade externa à unidade de saúde mais desenvolvida pelas equipes de saúde. Ela se caracteriza por utilizar uma tecnologia leve, permitindo o cuidado à saúde de forma mais humana, acolhedora, estabelecendo laços de confiança entre os profissionais e os usuários, a família e a comunidade, ampliando o acesso da população às ações da Saúde em um dos pontos de sua rede de atenção: o domicílio, a unidade residencial de determinada família. ${ }^{5}$

Para que seja eficaz, é imprescindível que o profissional que irá praticar compreenda a estrutura familiar e a sua funcionalidade. Tem com finalidade realizar a orientação à família, coletar informações sobre a condição socioeconômica, e melhorar o relacionamento familiar com o profissional de saúde. A ESF deve estar capacitada para acolher a pessoa idosa e ajudá-la a realizar as atividades do dia, as quais possui dificuldade para serem realizadas sozinhas, como alimentar-se, banhar-se, vestir-se, mobilizar, deambular, ir ao banheiro e manter controle das necessidades fisiológicas.

Aequipe daEstratégia de Saúdeda Família deveser composta no mínimo por: 1 médico, preferencialmente da especialidade medicina de família e comunidade, enfermeiro, preferencialmente especialista nesta área; auxiliar e/ou técnico de enfermagem e agente comunitário de saúde (ACS). Podendo fazer parte da equipe o agente de combate às endemias (ACE) e os profissionais de saúde bucal: cirurgião-dentista, preferencialmente especialista nesta área, e auxiliar ou técnico em saúde. ${ }^{4}$ Espera-se que a equipe realize uma assistência humanizada, com acompanhamento e apoio domiciliar, com o objetivo de melhoras as condições de qualidade de vida da pessoa idosa.

Para o acontecimento da visita domiciliar, não se faz necessária a presença de toda a equipe, e pode ser realizada pelo médico, enfermeiro, técnico de Enfermagem e ACS, porém é o enfermeiro o grande responsável.

Além disso, busca a promoção da qualidade de 


\section{Atuação do enfermeiro na visita domiciliar}

O cuidar em enfermagem inclui a execução de procedimentos técnicos aliados à adoção de atitudes condizentes com princípios humanitários, que assegurem a manutenção do respeito, dignidade e responsabilidade entre as pessoas envolvidas na relação de cuidado. ${ }^{6}$

Na visita domiciliar, a presença do/a enfermeiro/a é imprescindível, pois trata-se significativamente de uma atenção diferenciada na arte de cuidar.

$\mathrm{O}$ fato de esta ser uma atividade extensa e externa realizada pelos profissionais de saúde, cabe ao enfermeiro, ao realizá-la: constatar, orientar, educar, fornecer, e acompanhar os casos clínicos através da consulta, instrumento legal de suas ações, capaz de proporcionar-lhe respaldo para atuar com embasamento científico.

Ao realizar o atendimento, pode-se perceber o fortalecimento e até mesmo a criação de um vínculo profissional-paciente, identificando os problemas apresentados pelo paciente e consequentemente pela família, de modo a poder ajudar ambos. O enfermeiro deve atuar de modo ético e legal, a fim de empoderar as famílias vulneráveis à lutarem pelos seus direitos de saúde. ${ }^{7}$

Assim, a compreender a especificidade da população idosa e deve facilitaro acesso ao atendimento à saúde, promover a educação em saúde sobre a população idosa para os profissionais que irão acompanhá-lo na visita domiciliar.

Além disso, o enfermeiro deve atuar de modo em realizar 4 tipos de ações na visita domiciliar: ${ }^{8}$

- Ações interacionais: em que deve haver uma relação de ajuda/interação, dar apoio afetivo, mental ou psicológico, fortalecer uma relação de confiança, diálogo e escuta com o paciente;

- Ações educacionais, em que deve orientar familiares, cuidadores e pacientes desenvolvendo estratégias de ensino;

- Ações assistenciais: em que deverá realizar a gestão ou infusão de medicamentos, cuidado com feridas, avaliação de riscos, prevenção e complicações e procedimentos técnicos, como avaliação física, higiene pessoal, verificação de sinais vitais, cuidados de decúbito, exercícios para deambulação, atendimentos em casos de emergência, sondagens vesicais, sondagens gástricas e enterais, entre outros procedimentos privativos;

- Ações administrativas como planejamento e organização das visitas domiciliares e coordenação do cuidado.

A enfermagem necessita buscar o aprimoramento quanto à assistência, buscando um cuidado individual e personalizado para que exista um profissional com esse pensamento ampliado, dominando todos os programas de assistência à saúde na rede básica por ser uma área de primeiro contato à saúde. É necessário incluir na formação acadêmica uma metodologia problematize e que faça com que o acadêmico de enfermagem seja capaz de propor e executar mudanças no modelo de cuidar se tornando um enfermeiro crítico não só no âmbito hospital, mas também na rede básica de saúde, onde o cuidado é essencial, pois evita agravos à saúde e dar uma atenção ao idoso são uma tática de promover uma saúde para nossa população aumentando a qualidade de vida. ${ }^{9}$

\section{Vantagens da visita domiciliarecapacitação do enfermeiro para a sua realização}

O atendimento à saúde desenvolvido em domicílio favorece a promoção, proteção e a recuperação da saúde do idoso e da família, sendo um cuidado individualizado, respeitando a individualidade do paciente idoso e suas limitações, de forma a não interferir em seus valores e crenças.

A mudança no hábito alimentar e o uso correto da medicação tornaram-se valorizados após a o atendimento domiciliar. Os idosos passam a consumir alimentos com menos carboidratos e gorduras, além da inclusão de outros constituintes alimentares na composição de uma dieta saudável, como saladas e frutas e o aumento do consumo de água, que são hábitos importantes para que sejam evitadas a desidratação e iatrogenias. ${ }^{10}$

Além disso, há uma melhora na prática de atividades físicas e um maior cuidado quanto aos fatores de risco que possam existir no ambiente em que a pessoa idosa vive, sendo: objetos que ficam no caminho facilitando a queda em domicílio, como tapetes, móveis no caminho e outros utensílios.

\section{Conclusão}

A partir do presente estudo, pode-se conluir que o enfermeiro possui um grande papel na atuação da assistência à saúde e qualidade de vida da pessoa idosa. Ao realizar a visita domiciliar, o profissional de Enfermagem proporciona a criação de um laço profissional-paciente e identifica os problemas apresentados pelo cliente e consequentemente pela família. Desse modo, é de grande importância que enfermeiro esteja capacitado para realização da VD, afim de evitar os agravos em sua saúde e prestar uma assistência qualificada. Além disso, vale mencionar que através da visita domiciliar, é promovida a autonomia dos idosos, preservando a sua dignidade e integralidade, ao elevar a sua qualidade de vida. 


\section{Referências}

1. Brasil, MS. Departamento de Atenção Básica. Envelhecimento e saúde da pessoa idosa. Brasília: Ministério da Saúde, 2006. 192 p

2. Ferreira AMO. Visita domiciliar realizada pelo/a enfermeiro/a com enfoque na funcionalidade global da pessoa idosa: um estudo misto. [Dissertação]. Universidade Federal Fluminense, Niterói; 2019. 110p. Disponível em: $<$ https://pesquisa.bvsalud.org/enfermeria/resource/pt/biblio988027>

3. Wanderley RMM, Felisberto AMS et al. Avaliação da condição de saúde da população idosa na atenção básica. Rev enferm UFPE online. Recife. 2019 jan; 13(1): 472-82.

4. Brasil. MS. Política Nacional de Atenção Básica. Brasília: Ministério da Saúde. 2012; 112p.

5. Andrade AM, Guimarães AMDN, et al. Visita domiciliar: validação de um instrumento para registro e acompanhamento dos indivíduos e das famílias. Epidemiol. Serv. Saúde, Brasília. 2014 jan-mar; 23(1):165-175.

6. Santos FPA, Acioli S, Rodrigues VP, Machado JC, Souza MS, Couto TA. Práticas de cuidado da enfermeira na Estratégia Saúde da Família. Rev Bras Enferm [Internet]. 2016; 69(6):1060-7. Disponível em: <https://www. scielo.br/scielo.php?script=sci_arttext\&pid=S0034-71672016000601124>

7. Gomes MFP, Fracolli LA, et al; Atenção domiciliar do enfermeiro na Estratégia de Saúde da Família. O Mundo da Saúde, São Paulo - 2015 nov; 39(4):470-475. Disponível em: < https://pesquisa.bvsalud.org/bvsms/ resource/pt/mis-37762>

8. Coren. Manual de orientações para os profissionais de enfermagem de home care e cooperativas prestadores de serviços na assistência domiciliar do distrito federal. Brasília Coren-DF. 2019; 30p.

9. Ribeiro WA, et al. Processo de envelhecimento do idoso e a protagonização do enfermeiro na visita domiciliar na atenção primária de saúde. Revista Pró-UniverSUS [Internet]. 2019; 10 (2): 53-58. Disponível em: <http://editora.universidadedevassouras.edu.br/index.php/RPU/article/ view/2014>

10. Nogueira IS, Previato GF, Scolari GAS, Gomes ACO, Carreira L, Baldissera VDA. Intervenção domiciliar como ferramenta para o cuidado de enfermagem: avaliação da satisfação de idosos. Rev Gaúcha Enferm. 2016; 37. Disponível em: <https://www.scielo.br/scielo.php?script=sci arttext\&pid=S1983-4472016000500409> 